\title{
Inspection of the Integrity of a Multi-Bolt Robotic Arm Using a Scanning Laser Vibrometer and Implementing the Surface Response to Excitation Method (SuRE)
}

\author{
Hadi Fekrmandi ${ }^{1}$, Javier Rojas ${ }^{2}$, Jason Campbell ${ }^{3}$, Ibrahim Nur Tansel ${ }^{4}$, Bulent Kaya ${ }^{5}$, Sezai Taskin ${ }^{6}$ \\ ${ }^{1,2,3,4}$ Department of Mechanics and Materials Engineering, Florida International University, Miami, FL, 3374, U.S.A \\ hfekr001@fiu.edu.edu \\ jroja016@fiu.edu.edu \\ jcamp131@fiu.edu \\ tanseli@fiu.edu \\ ${ }^{5}$ Department of Industrial Design Engineering, Erciyes University, Kayseri, Turkey \\ bulentkaya@erciyes.edu.tr \\ ${ }^{6}$ Department of Electrical and Electronics Engineering, Celal Bayar University, Manisa, Turkey \\ sezai.taskin@cbu.edu.tr
}

\begin{abstract}
The integrity of a robotic arm was examined remotely via a scanning laser vibrometer (SLV) in order to detect loose bolts. A piezoelectric element (PZT) was bonded on the robot arm for excitation of surface guided waves. A spectrum analyzer generated surface waves within the 20$100 \mathrm{kHz}$ range. The propagation of the waves was monitored with the SLV at the programmed grid points on the robot arm.

The surface response to excitation (SuRE) method was used to calculate the spectrums of the signals, and compare the reference scan with the altered scan. Comparisons of before and after the scan showed that after loosening the bolt on the robot arm, spectrums of all the grid points changed to some extent, however, the largest changes occurred in the vicinity of the loosened bolts.

The study shows that the SuRE method was capable of detecting the presence and location of loosening bolts using only one PZT element on a complex structure. There are two most important advantages of the SuRE method over the widely used impedance-based technique. The first advantage is the elimination of an expensive impedance analyzer; the second advantage is remotely monitoring capability as long as the surface is excited properly.

Hadi Fekrmandi et al. This is an open-access article distributed under the terms of the Creative Commons Attribution 3.0 United States License, which permits unrestricted use, distribution, and reproduction in any medium, provided the original author and source are credited.
\end{abstract}

\section{INTRODUCTION}

Many structural health monitoring (SHM) techniques are developed to improve the reliability and safety of the systems while the maintenance costs and service time are reduced. Some of these methods are developed for detection of the loose bolts. Bolt joints have been widely used in many civil, mechanical and aero-space structures. In some critical operations, any failure could have catastrophic consequences and manual inspection of the bolts is not feasible. The development of remote monitoring techniques is necessary to address these applications. In this study, the integrity of bolted plates of a robot was inspected by implementation of the surface response to the excitation (SuRE) method.

Todd et al. (Todd, Nichols, Nichols and Virgin 2004) evaluated the condition of bolt joints by using the modal parameters. They experimentally found that the modal properties were relatively insensitive to the clamping force as long as they were above a critical level. They suggested evaluating the modal parameters may not be a reliable approach for assessing the condition of the bolt joints, and alternative methods should be considered for developing more sensitive monitoring techniques.

Recently, active SHM methods have received a lot of attention. These methods detected structural defects via exciting high-frequency surface waves on the target structure and monitoring the response. Guided Lamb-wavebased methods ( $\mathrm{Su}, \mathrm{Ye} \& \mathrm{Lu}, 2006)$ (Raghavan \& Cesnik, 
2007) and electromechanical impedance (EMI)-based methods (Yan \& Chen, 2010) (Annamdas \& Radhika 2013) are two examples of this trend.

Peairs et al. (Peairs, Park and Inman 2004) employed the impedance-based structural health monitoring technique by applying high-frequency excitations through a PZT transducer. They found that the impedance characteristics were sensitive to loosening bolts. They also tried to replace the impedance analyzer with a smaller and more effective device, since it was heavy and bulky. Ritdumrongkul et al. (Ritdumrongkul, Abe, Fujino and Miyashita 2004) studied the structural integrity of two aluminum beams connected through a bolted joint. They found the EMI method promising; however, they mentioned that the application of the method to real structures required further study of the characteristics of more complex structures and environmental effects. Chakraborty et al. (Chakraborty, Kovvali, Wei, Suppappola, Cochran and Chattopadhyay 2009) introduced an advanced time-frequency signal processing technique for detecting loose bolts in complex structures. Their method required a significant amount of experimental data for the training of the damage classification algorithm. They suggested use of numerical simulation for preparation of training data.

In order to overcome the complexity and costs of the EMI method, Tansel and co-workers (Tansel, Singh, Korla, Grisso, Salvino, \& Uragun, 2011) developed the SuRE method. SuRE evaluated the surface-response characteristics similar to the EMI, but required simpler instrumentation. The SuRE method used a piezoelectric element to excite the surface with a sweep sine wave, and the surface response was evaluated with another sensor. Fekrmandi et al. (Fekrmandi, Rojas, Wolff, Tansel Gonzalez and Uragun 2013) used SLV as an alternative non-contact sensor to the network of PZT elements to identify the location of applied load on a beam structure.

SLV was used in an effort to detect fatigue cracks using high frequency guided waves (Leong, Staszewski, Lee, \& Scarpa, 2005). In their study, the lamb wave method was used. Previous studies have proven that frequency domain methods have considerably better performance for health monitoring of bolted joints like gas pipelines and composite structures, (Peairs, Park, \& Inman, 2004). They could estimate the location of the loose bolts only if multiple elements were installed carefully. In critical applications like integrity of bolt joints in satellites (Arritt et al. 2008), it was hard to access and impractical to create a large network of PZT sensors. The motivation behind this study is to introduce SLV as an alternative to sensor networks. The authors believe in many critical applications, such as the inspection of the control surfaces of rockets at the launch pad, the use of SLV could be easily justified.

The frequency domain methods have not been implemented through non-contact sensors for health assessment of multi- bolt joints on a robot arm structure. In this study, SuRE was implemented to assess data from various scanning points for estimating the location of one or more loose bolts on the robot structure.

\section{THEORETICAL BACKGROUND}

Liang et al. (Liang, Sun, \& Rogers, 1994) first developed a one degree of freedom model for a coupled electromechanical PZT actuator system. Their study showed that the change of the structural impedance would dominate the electrical impedance of the PZT. Monitoring the impedance of the PZT with an impedance analyzer was enough to determine the condition of the structure. To evaluate the condition of the structure, the real part of the impedance was analyzed, since it was more sensitive to structural damages (Bhalla, Naidu \& Soh, 2003). The SuRE method monitors the surface response to excitation by using a separate actuator and sensor(s). The characteristics of SuRE and EMI are very similar.

In this study, the structure was excited using a piezoelectric element and the surface vibrations were monitored by the scanning laser vibrometer. The reference scan was performed when all the bolts were tight. The altered scans were performed after one or more bolts were loosened. The change of the compressive forces on the structure surfaces changed the surface response to excitation. To quantify the change, the Squared Difference (SD) of two matrices is calculated with the following equation:

$$
D^{2}{ }_{i, j}=\left(A_{i, j}-R_{i, j}\right)^{2}
$$

$\mathbf{R}$ and $\mathbf{A}$ are the reference and altered data matrices obtained by the spectrum analyzer. Data matrices have $m$ rows by $n$ columns, where each column includes the frequency spectrums of a scan point. The frequency range was distributed over the matrix row. The average of the squared differences was calculated with the following equation:

$$
\text { Average }=\frac{1}{m n} \sum_{i=1}^{m} \sum_{j=1}^{n} D_{i, j}^{2}
$$

where $m$ and $n$ are the number of frequencies and the number of scan points, respectively. The Normalized Squared Differences (NSD) was obtained from dividing the squared differences by the average:

$$
\bar{D}_{i, j}=\frac{D_{i, j}^{2}}{\text { Average }}
$$

The normalized differences for each scan point could be averaged versus the frequency range to obtain the Normalized Sum of Squared Differences (NSSD), and in fact this criterion was an array with the size of scan points:

$$
\bar{S}_{i}=\frac{1}{n} \sum_{j=1}^{n} \bar{D}_{i, j}
$$


$\bar{S}$ is a matrix with the size of the scanning grid that contains a normalized value for each scanning point. This normalized value quantitatively represents the amount of change in the spectrum for each scanning point. Depending on the dimension of the scan grid, $\bar{S}$ could be a one-dimensional or two-dimensional array and could be represented in different graphical ways.

\section{EXPERIMENTAL SETUP}

The surface vibrations were measured at the programmed grid points by using the PolyTech PSV-400 SLV (Figure 1). The Stanford Research Systems SR780 2-channel network signal analyzer (Figure 2) generated the excitation signal and monitored the signals coming from the SLV. A power amplifier magnified the signal of the signal analyzer before it was given to the PZT. The signal analyzer calculated the spectrum after the signal was digitized.

The analog sensors of the laser head itself can monitor the vibrations well above $100 \mathrm{kHz}$, but the vibrometer controller analog-to-digital converter sampling frequency peaks at 100 $\mathrm{kHz}$. This caps the maximum frequency that the laser software can scan at $40 \mathrm{kHz}$. To overcome this limitation, the Stanford signal analyzer with a maximum A/D conversion rate of $250 \mathrm{kHz}$ was connected to the laser head. This allows us to capture frequencies up to $100 \mathrm{kHz}$.

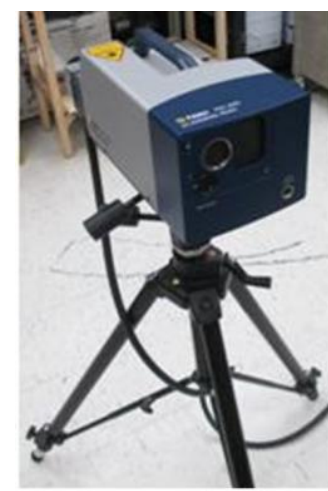

(a)

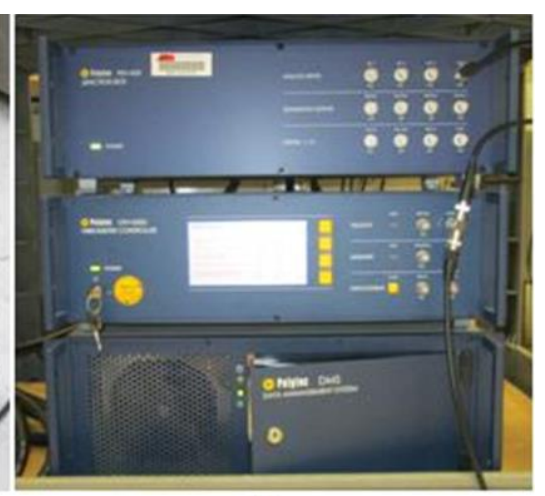

(b)
Figure 1. PolyTech PSV-400 Scanning Laser Vibrometer (a) Laser head (b) Vibrometer controller and junction box

The laser system can operate for long periods of time and conduct scans at multiple points in a relatively short period of time. The signal analyzer can only scan one point at a time. During the experiment, the operator manually selected points with the laser and performed scans with the signal analyzer. The low and high ends of the sweep sine wave signal were selected as $20 \mathrm{kHz}$ and $100 \mathrm{kHz}$, respectively. The low end was selected to prevent audible sounds in the lab. The maximum sampling frequency of the signal analyzer's A/D determined the high end.

The dimensions of the aluminum robotic control arm were $152.5(\mathrm{~cm}) \times 47(\mathrm{~cm}) \times 33(\mathrm{~cm})$ (Figure 3). A $3 / 4$ " diameter APC PZT model D-.750"-2MHz-850 WFB was attached to the center of the scanned area where the bolts were located. The dimensions of the scanned plate were: $78.75(\mathrm{~cm}) \times$ $7.62(\mathrm{~cm})$ with a $1.27(\mathrm{~cm})$ thickness and the bolts were located approximately $7.62(\mathrm{~cm}) \times 4.45(\mathrm{~cm})$ apart from each other. 18 bolts were used for the structure.

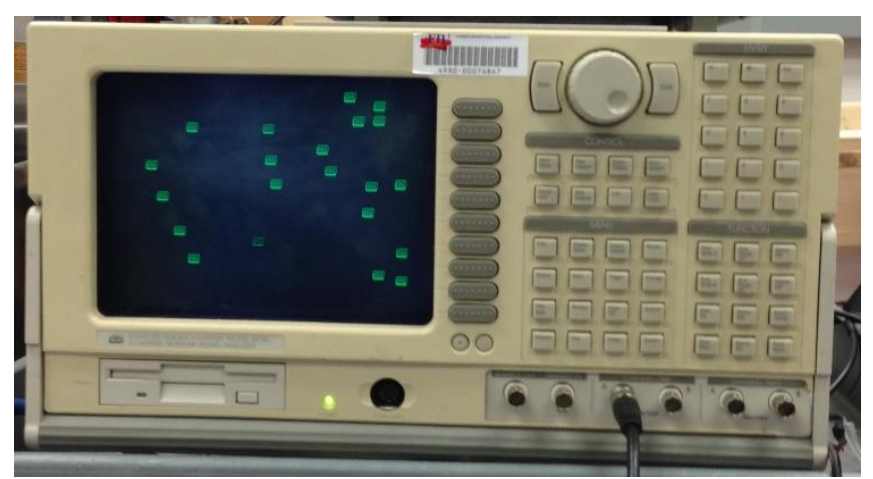

Figure 2. Stanford Research Systems SR780 2-Channel Network Signal Analyzer

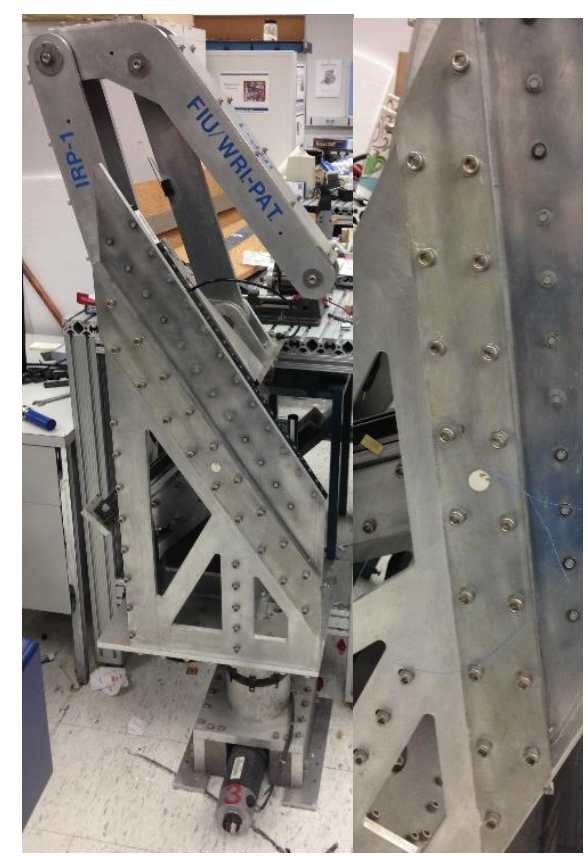

Figure 3. The robot structure and close up view of the scanned section

The SLV was located five feet away from the control arm of the robot (Figure. 4). The reference data was collected when all bolts were tight by scanning the vibration at the points on the grid. Then, one bolt or multiple bolts were loosened and another scan (altered data) was measured using the same grid points. Finally, these scans were compared to each other using the algorithm described in the theoretical background. The positions of loose bolts were identified by locating the scan points with the highest NSSD values. 


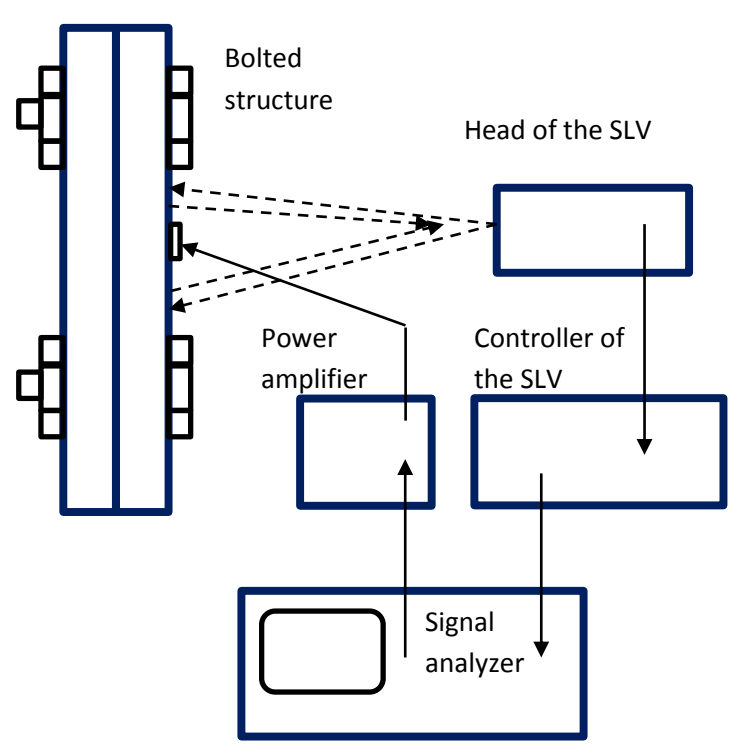

Figure 4. Experimental schematic

\section{RESULTS}

The tests were performed on a robot arm with a bolted joint that was connected to the main structure via 18 bolts and nuts, out of which 16 were considered for this study (Figure. $5)$.

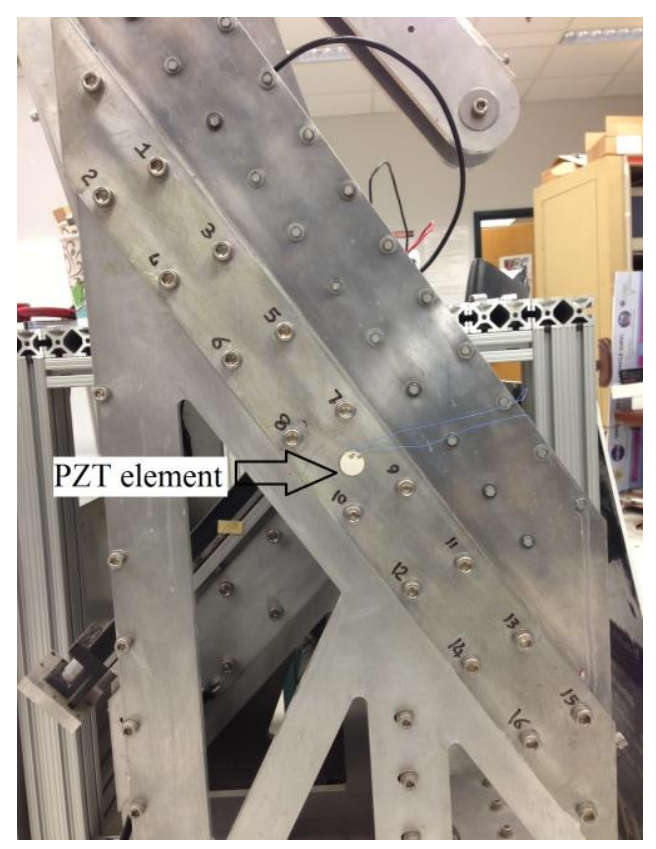

Figure 5. Bolted joint on the robot arm with bolt numbers

\subsection{Natural Frequencies}

The simple bump test method (Scheffer, \& Girdhar, 2004) was used to determine the natural frequencies of the robot arm. The structure was hit with an impact hammer and the signal was collected from the PZT element on the arm. The Fast Fourier Transform (FFT) of the signal was calculated. The dominant peaks, which were determined by peak hold of the instant FFT response, were used to determine the natural frequencies of the structure. Table 1 shows the first five natural frequencies of the robot arm in two separate tests of the tight and loose bolt cases:

Table 1. Natural frequencies of the robot arm

\begin{tabular}{|c|c|c|c|c|c|}
\hline & $1^{\text {st }}$ & $2^{\text {nd }}$ & $3^{\text {rd }}$ & $4^{\text {th }}$ & $5^{\text {th }}$ \\
\hline Tight & 61.35 & 82.39 & 180.04 & 381.46 & 628.61 \\
Bolt & $(\mathrm{Hz})$ & $(\mathrm{Hz})$ & $(\mathrm{Hz})$ & $(\mathrm{Hz})$ & $(\mathrm{Hz})$ \\
\hline Loose & 61.35 & 82.39 & 180.05 & 381.47 & 628.62 \\
Bolt & $(\mathrm{Hz})$ & $(\mathrm{Hz})$ & $(\mathrm{Hz})$ & $(\mathrm{Hz})$ & $(\mathrm{Hz})$ \\
\hline
\end{tabular}

To test the effect of the loose bolts on the natural frequencies of the structure, the measurements were repeated after loosening the single and multiple bolts. The changes of the natural frequencies were very small. In the experimental studies, such small changes have been observed when the experiments were repeated at the same conditions. Environmental noise and small computational errors of signal analyzers could cause those changes. Our results agreed with the previous studies like Todd et al. (Todd, Nichols, Nichols and Virgin 2004), where the natural frequencies did not exhibit enough sensitivity for detection of loose bolts.

In our study, high frequency surface waves were employed and the changes in the measured spectrums were used for detection of the single and multi-loose bolts similar to the impedance method.

\subsection{Detection of Loose Bolts in Linear Grid}

The first experiment was designed to identify the effect of the presence of a single loose bolt on the measured spectrum. First, a single scanning point was chosen near the location of bolt 10 . The initial reference scan was measured when all the bolts were tight. The reference scan measurement was repeated before loosening any bolt to evaluate the consistency of test characteristics. The altered scan data was collected for after the bolt was loosened.

The spectrums resulting from the first two scans were almost overlapping each other over the whole frequency range 20-100 kHz. This can be observed in Figure. 6(a). This observation demonstrated consistency of the data collection and analysis system. The third spectrum was very different than the first two (Figure. 6(b)). The propagation characteristic of the surface waves was consistent as long as the bolt was tightened. The propagation characteristic drastically changed when the bolt was loosened. The 
impedance method detects the presence of loose bolts by monitoring the same characteristics of the impedance spectrum.

(a)

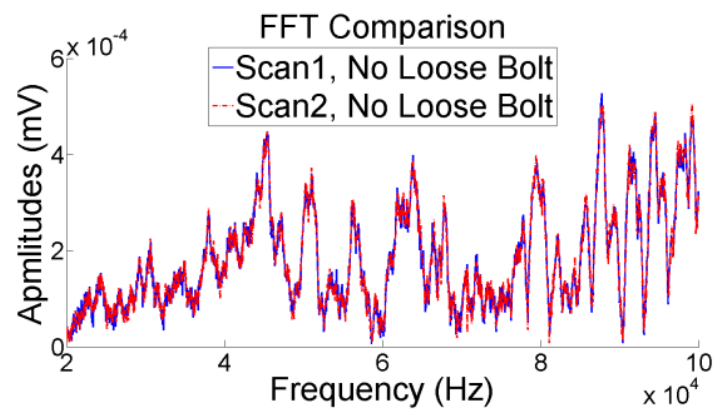

(b)

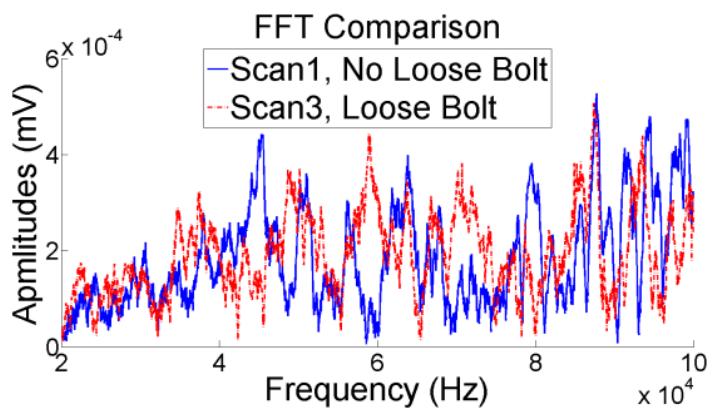

Figure 6. FFT spectrum comparison

(a) Scan1 with all bolts tight and Scan 2 with all bolts tight

(b) Scan 1 with all bolts tight and Scan 3 with a loose bolt

The next scan was performed over a linear grid including four bolts in a row. The bolts 4, 6, 8 and 10 in Figure 7 were considered. After the reference scan, only bolt 8 was loosened. For each bolt, two scanning points were specified on the grid. These points were located on the left and righthand side of the bolts (Figure 7).

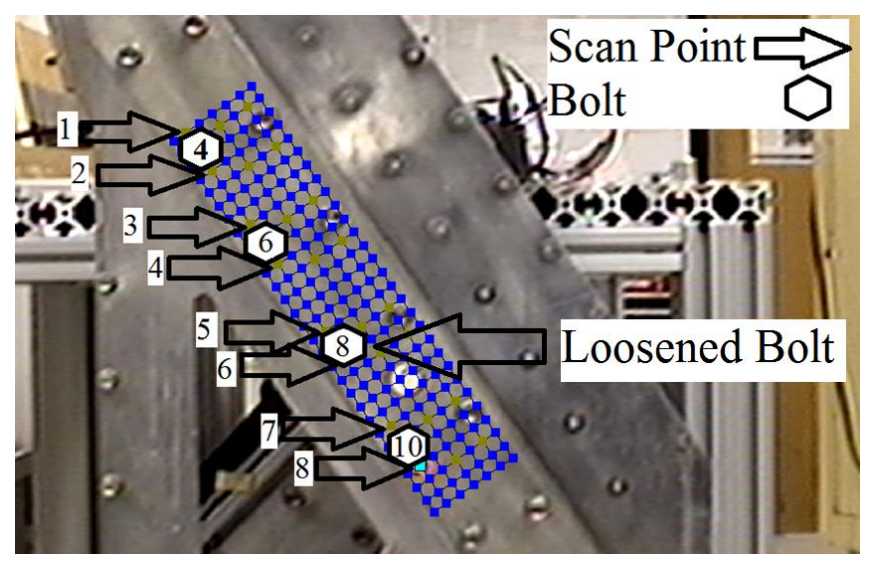

Figure 7. Local linear scan grid with eight scanning points specified on both sides of the lower four bolts.
Based on the algorithm developed on the theory section of this paper, the normalized sum of squared differences (NSSD) was calculated from the spectrums before and after loosening bolt 8, which was located between scan points number 5 and 6 in Figure 7. Figure 8 compares the NSSDs for bolt 8 when it was tight versus loose.

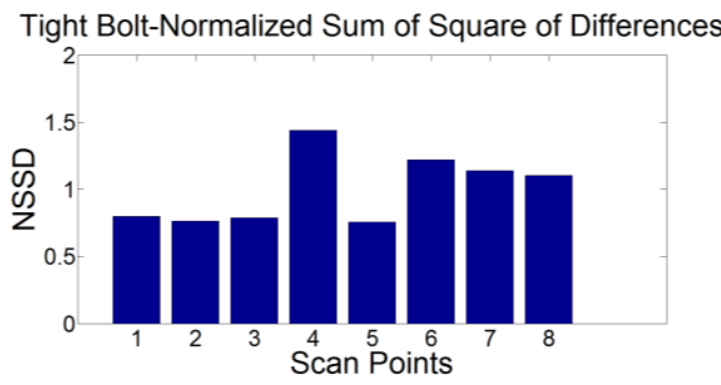

Loose Bolt-Normalized Sum of Square of Differences

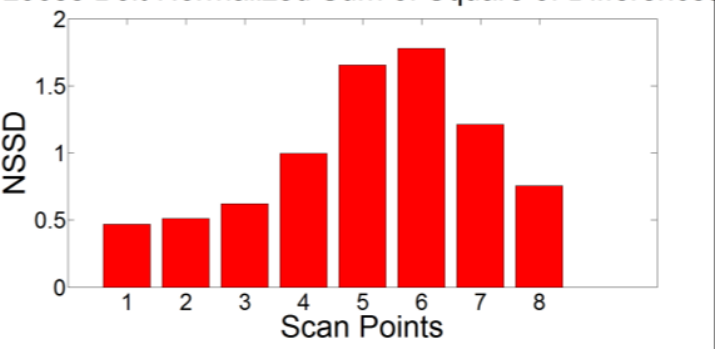

Figure 8. Normalized sum of square of differences for linear local scan for (a) before (b) after; single bolt was loosened

Figure. 8(b) was used for estimating the location of the loose bolt. The NSSD of the scanning points 5 and 6 were very high compared to the other scan points. Since the scan points 5 and 6 are located by bolt 8 , this result clearly indicates that bolt 8 was loosened. The results of this section also confirm that the highest values of the NSSDs are observed at the nearest scanning points to the loosened bolt.

The typical changes of the SSD values of a structure without any loose bolts and with one loose bolt are presented in Figure 9. The SSD values of the scan points are due to measurement errors, external noise and calculation errors. These values were close to each other when there were no loose bolts. The SSD values of all scan points increased more than $70 \%$ when a bolt loosened. A threshold value may be found easily since the change was significant. The change of the SSD at the scan point closest to the loose bolt was more than $150 \%$. Based on this figure, the SSD values may be used for detection of the loose bolt and estimation of its location. Using the normalized SSD (NSSD) values are more convenient for location of a loose bolt while the existence of the problem is detected from the SSD values. 


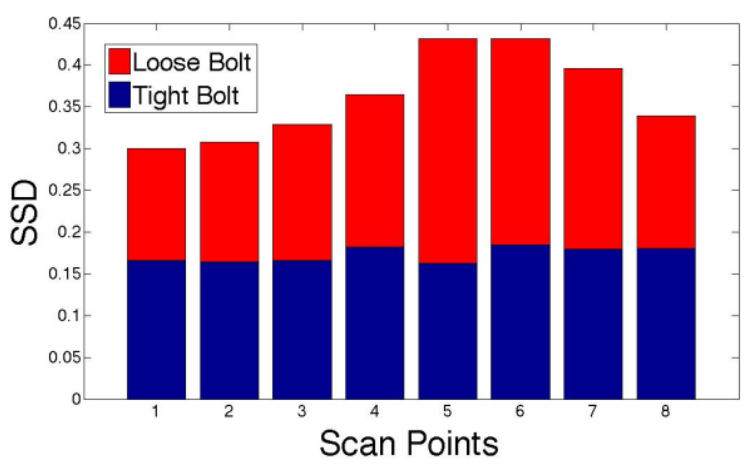

Figure 9. Sum of Squared Difference (SSD) values before and after loosening bolt 8 .

The proposed method may be implemented in the field by using the following procedure: First, the SSD values of the scan points are calculated. If the SSD values are below a threshold, the structure should not have any loose bolts. To improve the robustness of the method, the average of the SSD values are calculated and a secondary threshold is established by multiplying the average SSD value with a coefficient such as 1.2. The secondary threshold may be compared with the SSD values of the scan points to avoid false alarms if the diagnostic system is used in extremely different operating conditions. Such as testing the system when the engines are at the idle and cruise speeds. The second step will be used when some of the SSD values are over the threshold. The location of the loose bolt will be estimated by using the NSSD values.

\subsection{Detection of Loose Bolts in Two Dimensional Grid}

The two-dimensional grid was designed to locate one scan point next to each bolt. Therefore, the number of scanned points and the number of bolts on the structure were the same. The reference scan and a second scan with the same conditions were performed to confirm the consistency of the results. Figure 10 shows the normalized sum of squared differences when both scans were measured before loosening the bolt (first and second reference scans). There are 16 bars and each one corresponds to a point close to one of the bolts.

\section{Normalized Sum of Square of Differences}

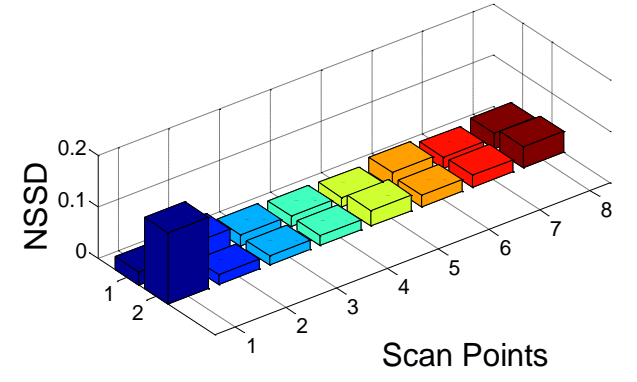

Figure 10. Bar diagram of normalized sum of square of differences for full arm without any loose bolts
In Figure 10, no major changes were identified for scan points before loosening the bolt. Another scan was performed after loosening two bolts in the upper row (bolts 3 and 5). Figure 11 shows that the approximate location of these bolts could be detected.

\section{Normalized Sum of Square of Differences}

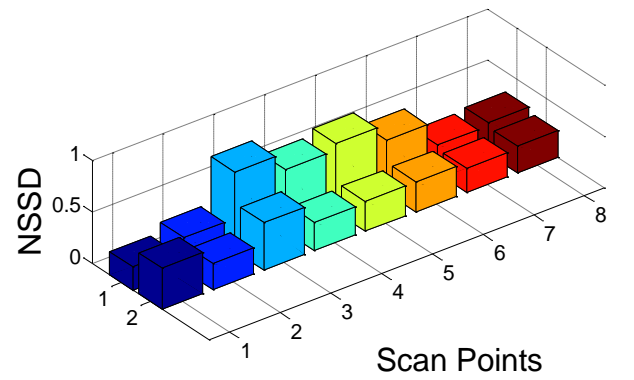

Figure 11. Bar diagram of normalized sum of squared differences for full arm with two loose bolts

The test was repeated after the bolts close to the scanning points 3 and 5 were loosened. The contour map of Figure 12 shows that the highest peaks are at the scanning points close to the location of the loose bolts. The adjacent areas were also affected. The contour map shows that the highest peaks were located at columns 3 and 5, and identified the loose bolts better. The contour map was not smooth since the numbers of the grid points were very limited.

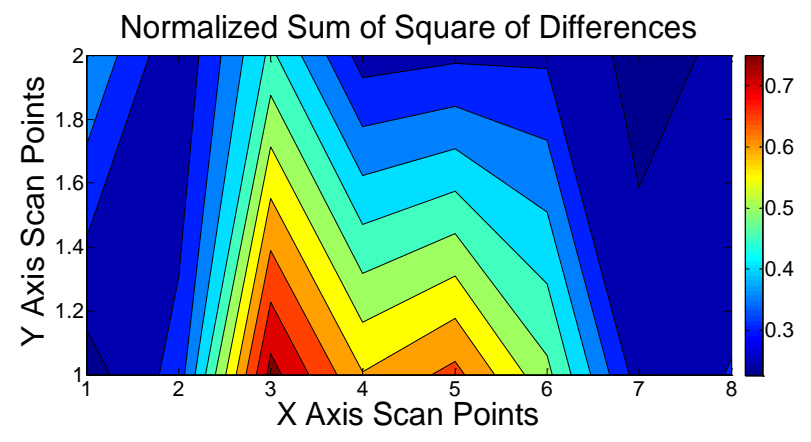

Figure 12. Contour map of normalized sum of squared differences for full arm scans with two loose bolts; bolt 3 and bolt 5 are loosened.

\subsection{Detection of Loose Bolts in Full Arm Grid}

In this section, an experiment for a single loose bolt was performed, followed by another experiment using multiple loose bolts. The density and size of the scan grid were designed to cover the whole bolted section of the robot arm.

\subsubsection{Detection of Single Loose Bolt in Full Arm Grid}

The size of the scan grid was increased to improve the resolution and to demonstrate the consistency of the results. 
The vibrations of 60 points were monitored on a $20 \times 3$ scan grid (Figure 13).

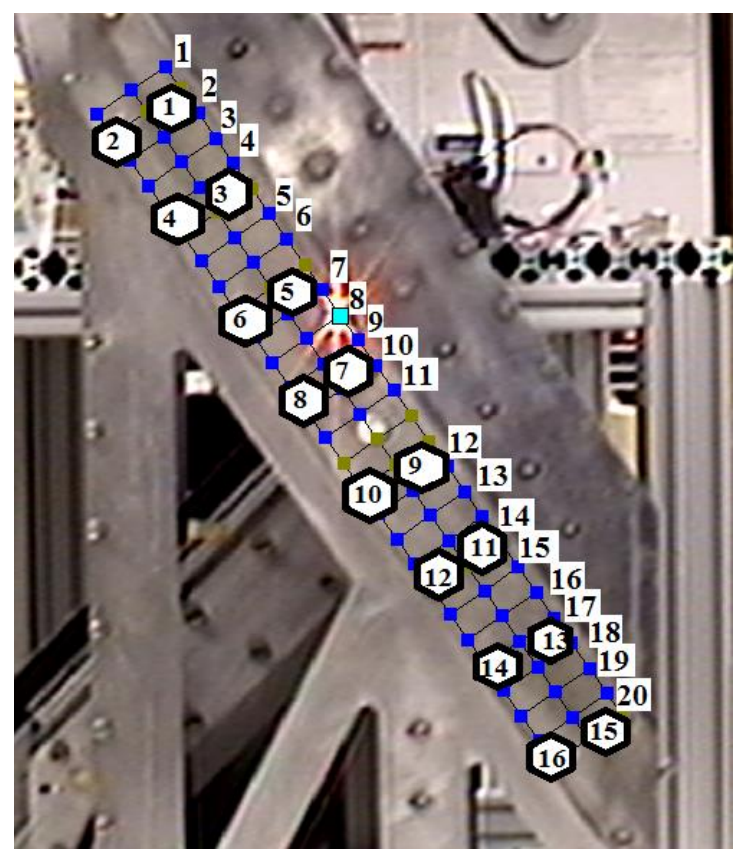

Figure 13. Two-dimensional full arm scan grid with 20 columns and 3 scan points in each column

Data was not collected from the scan points that were located on the piezoelectric actuator. The scan test points are illustrated with the blue points. Figure 14 shows a threedimensional bar diagram that demonstrates the normalized sum of the squared differences. In this experiment, bolt 8 was loosened.

\section{Normalized Sum of Square of Differences}

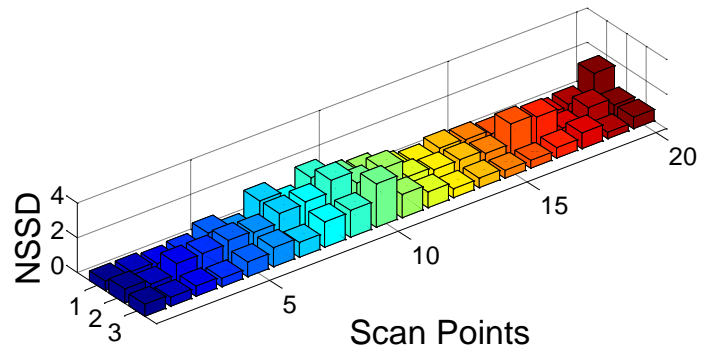

Figure 14. Normalized sum of squared differences for full arm scan; only bolt 8 is loosened

The maximum values of NSSDs are located around columns 9 and 10 in Figure 14. In Figure 13, columns 9 and 10 are encircling bolt 8 . This means that the location of the maximum NSSD values correctly identified the loosened bolt 8 .
The two-dimensional contour map in Figure 15 is also prepared using the same NSSD values, where the location of the loose bolt is better identified.

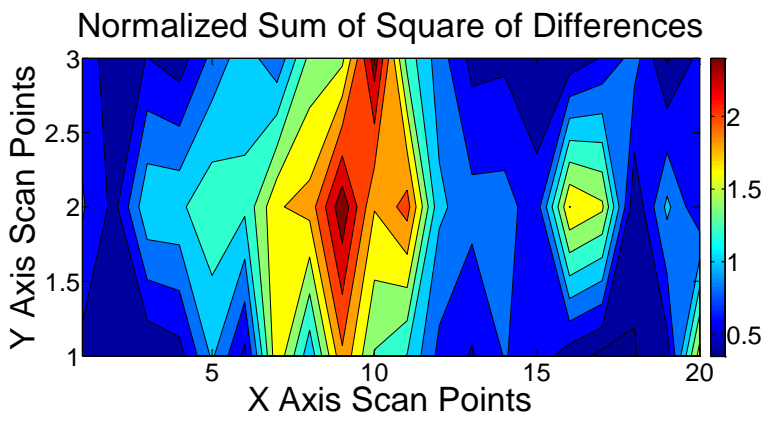

Figure 15. Contour map of normalized sum of squared differences for loosening bolt 8

The red spot in Figure 15 shows the peak of the NSSD values that corresponds to the scan points 9 and 10 .

\subsubsection{Detection of Multiple Loose Bolts in Full Arm Grid}

The reference data was collected when all bolts were tightened. The same $20 \times 3$ scan grid in Figure 13 was used in this experiment. To evaluate the performance of the proposed approach in a more challenging scenario, bolts 2 and 7 located at opposite corners of left side of the scan grid were loosened. The normalized sums of squared differences were calculated and those values were used to prepare the 3D bar graph and 2D contour map.

\section{Normalized Sum of Square of Differences}

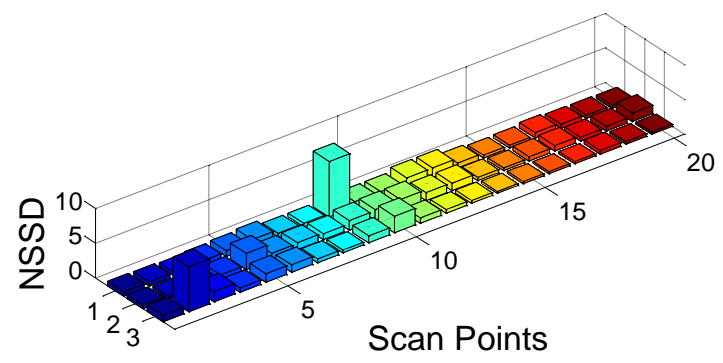

Figure 16. Normalized sum of squared differences for full arm scan; bolt 2 and bolt 7 are loosened

Figure 16 shows the NSSD values when bolts 2 and 7 were loosened. The highest peaks in this bar graph were located at the lower $2^{\text {nd }}$ column and upper $9^{\text {th }}$ column of the scan grid. These scan points are adjacent to the bolts 2 and 7 in Figure 13.

The contour map of the NSSD values presented in Figure 17 clearly illustrates the location of the loose bolts within two red spots. 


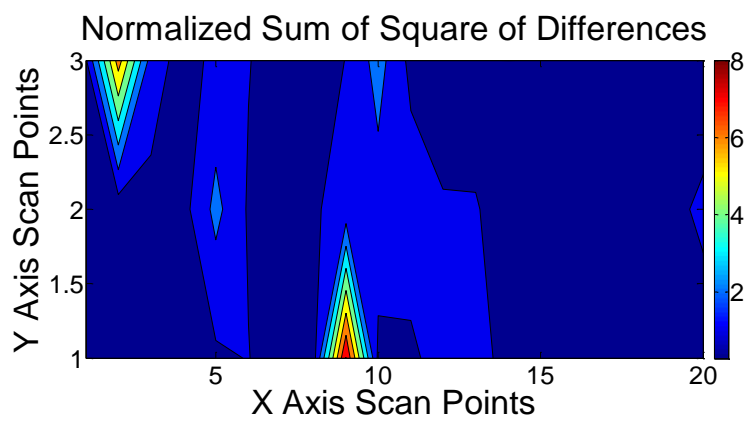

Figure 17. Contour map of the normalized sum of square of differences for multiple bolt loosening

The spectrums of each point were very similar when the test was performed using the same test conditions. When one or multiple bolts were loosened the propagation characteristics of the surface waves were changed. The changes of the spectrums were consistently more significant around the loosened bolts. The location of the loose bolts could be easily visualized with the help of 3-D bar graphs and 2-D contour maps. The results improved by increasing the grid points. The optimal grid size depends on the geometry and material of the structure, and may be determined with several experiments.

Here, it is noteworthy to emphasize the importance of attention to the noise problem during the experiments. Since the signal analyzer uses the peak hold to capture the frequency spectrum, large oscillations in the input signal during the capturing process could influence the peak holds and change their original values. During the data acquisition process, operator must constantly monitor the spectrum on the signal analyzer screen. In the case of any out-of-focus operation, the level of noise in the spectrum increases instantly and eats up the lower parts of the frequency spectrum throughout the entire frequency spectrum (Figure 18). Once the spectrum is distorted due to noise, it will no longer overlap the intact frequency spectrum from the same scan point. This way the comparison algorithm of SuRE method will calculate an increased NSSD value for that point and system will generate a false alarm.

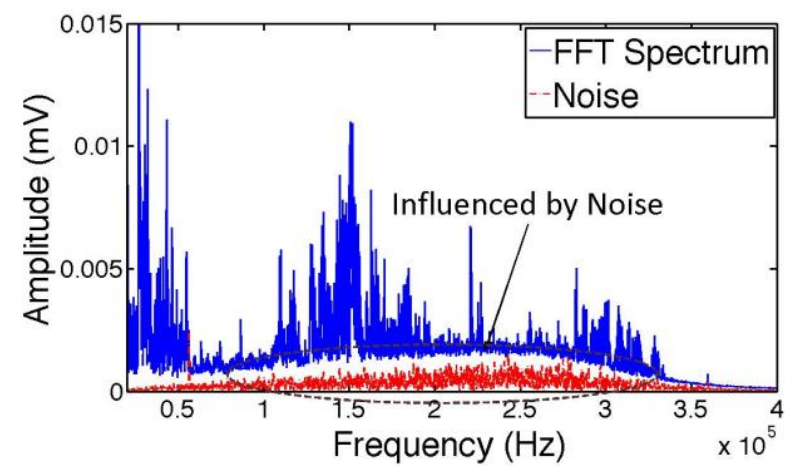

Figure 18. Effect of noise in the FFT spectrum

\section{CONCLUSION}

The surface response to the excitation (SuRE) method was used to identify the loose bolts on a large robot structure. The surface was excited with a piezoelectric element and the response was monitored remotely with a scanning laser vibrometer.

The loose bolts were identified accurately at all tests; either single bolt or multiple bolts were loosened. The surface vibrations were very small and required careful data collection with a separate spectrum analyzer. However, the SuRE method was found effective for detecting the loose bolts and identifying their location. The scanning laser vibrometer was able to collect the data very quickly and test many points on the structure without attaching a large number of piezoelectric elements at the considered grid location. In addition, the scanned surface area under the laser beam was extremely small and convenient for special applications involving miniature machine components.

The study was performed with different scan grid sizes to investigate the effect of the grid size in the results. It was necessary to have at least one scanning point next to each bolt to be able to locate the loose bolt(s). The scan grid with the minimum grid points required the minimum scanning time. Increasing the number of the grid points proportionally increased the scanning time while the resolution improved, and consequently the locations of the loose bolts were identified more accurately. The 3-D bar graphs and 2-D contour maps helped the visualization of the location of the loose bolts. Among them, 2-D contour maps were more effective when the number of grid points were satisfactory.

The major challenge of this study was the high noise to signal ratio. The small amplitudes of the surface waves and remote measurement of these tiny surface oscillations were two primary sources of the noise. The surface waves were created with a PZT element attached to the surface. To maximize the amplitude of the surface waves, a power amplifier was used to provide higher power to the PZT element than the spectrum analyzer can provide. However, at the $20-100 \mathrm{kHz}$ frequency range, the amplitudes of available exciters are much smaller compared to the typical electro-dynamic exciters operating at the $0-200 \mathrm{~Hz}$ frequency range. In addition, the amplitude of the waves decreases while the waves travel from the PZT to the boundaries.

The second source of noise was from scanning from a remote location to measure the tiny surface waves. Compared to monitoring the voltage coming from the PZTs attached to the surface, the noise is much higher at the SLV outputs. This could be due to the noncontact nature of the measurement and very small scanning surface area. In addition, the power of the laser beam was lower at the boundaries of the scanned grid compared to the central point. The auto focusing capability of the SLV avoided out- 
of-focus operation of the optic system at the boundaries. The size of the scan area should be carefully selected to be sure that the noise is within the acceptable levels when the boundaries are scanned. Therefore, there is a limitation in the dimension of the scan area depending on the distance of the laser head from the target area.

In practical applications, assessment of the environmental noise is recommended before implementing any SHM method. Generally, forced vibrations and their harmonics disappear within $20 \mathrm{kHz}$; however, impact forces are felt throughout the entire spectrum. If the environmental noise is very low at the test frequency range, the SuRE method may be implemented by using the SLV. Instead of the SLV, multiple piezoelectric elements may be attached to the grid points for implementing the SuRE method if the noise is too high for the SLV system. Authors believe the noise problem needs to be thoroughly investigated before implementing the proposed method at the field.

\section{ACKNOWLEDGEMENT}

The authors gratefully acknowledge the Army Research Office for funding the shared facilities used in this research at Florida International University. (Grant Number 58940RT-REP)

\section{NOMENCLATURE}

$\begin{array}{ll}\text { R } & \text { Reference Matrix } \\ \text { A } & \text { Altered Matrix } \\ \mathbf{D} & \text { Differences Matrix } \\ \overline{\boldsymbol{D}} & \text { Normalized Differences Matrix } \\ \overline{\boldsymbol{S}} & \text { Normalized Sum of squared differences }\end{array}$

\section{REFERENCES}

Annamdas, V. G., \& Radhika, M. A. (2013). Electromechanical impedance of piezoelectric transducers for monitoring metallic and non-metallic structures: A review of wired, wireless and energyharvesting methods. Journal of Intelligent Material Systems and Structures, 24(9), 1021-1042.

Arritt, B. J., Buckley, S. J., Ganley, J. M., Welsh, J. S., Henderson, B. K., Lyall, M. E., ... \& Roopnarine, R. (2008, March). Development of a satellite structural architecture for operationally responsive space. In The 15th International Symposium on: Smart Structures and Materials \& Nondestructive Evaluation and Health Monitoring (pp. 69300I-69300I). International Society for Optics and Photonics.

Bhalla, S., Naidu, A. S., \& Soh, C. K. (2003, October). Influence of structure-actuator interactions and temperature on piezoelectric mechatronic signatures for NDE. In Smart Materials, Structures, and Systems (pp. 263-269). International Society for Optics and Photonics.Biographies
Chakraborty, D., Kovvali, N., Wei, J., PapandreouSuppappola, A., Cochran, D., \& Chattopadhyay, A. (2009). Damage classification structural health monitoring in bolted structures using time-frequency techniques. Journal of Intelligent Material Systems and Structures, 20(11), 1289-1305.

Fekrmandi, H., Rojas, J., Wolff, M., Tansel, I. N., Gonzalez, S., and Uragun, B., (2013 November). Monitoring the Health of a Beam Remotely by using Scanning Laser Vibrometer. In proceedings of ASME District F Early Career Technical Conference (ECTC 2013).

Leong, W. H., Staszewski, W. J., Lee, B. C., \& Scarpa, F. (2005). Structural health monitoring using scanning laser vibrometry: III. Lamb waves for fatigue crack detection. Smart Materials and Structures, 14(6), 1387.

Liang, C., Sun, F. P., \& Rogers, C. A. (1994). Coupled electro-mechanical analysis of adaptive material systems-determination of the actuator power consumption and system energy transfer. Journal of Intelligent Material Systems and Structures, 5(1), 1220.

Peairs, D. M., Park, G., \& Inman, D. J. (2004). Improving accessibility of the impedance-based structural health monitoring method. Journal of Intelligent Material Systems and Structures, 15(2), 129-139.

Raghavan, A., \& Cesnik, C. E. (2007). Review of guidedwave structural health monitoring. Shock and Vibration Digest, 39(2), 91-116.

Ritdumrongkul, S., Abe, M., Fujino, Y., \& Miyashita, T. (2004). Quantitative health monitoring of bolted joints using a piezoceramic actuator-sensor. Smart Materials and Structures, 13(1), 20.

Scheffer, C., \& Girdhar, P. (2004). Practical machinery vibration analysis and predictive maintenance. Access Online via Elsevier.

Su, Z., Ye, L., \& Lu, Y. (2006). Guided Lamb waves for identification of damage in composite structures: A review. Journal of sound and vibration, 295(3), 753780.

Tansel, I. N., Singh, G., Korla, S., Grisso, B. L., Salvino, L. W., \& Uragun, B. (2011, June). Monitoring the integrity of machine assemblies by using surface response to excitation (SuRE) approach. In Recent Advances in Space Technologies (RAST), 2011 5th International Conference on (pp. 64-67). IEEETechnical Conference (ECTC), 2013 13th Annual Conference on (pp. 302-306, Vol.12). ASME

Todd, M. D., Nichols, J. M., Nichols, C. J., \& Virgin, L. N. (2004). An assessment of modal property effectiveness in detecting bolted joint degradation: theory and experiment. Journal of sound and vibration, 275(3), 1113-1126.

Yan, W., \& Chen, W. Q. (2010). Structural health monitoring using high-frequency electromechanical impedance signatures. Advances in Civil Engineering, 2010. 


\section{BIOGRAPHIES}
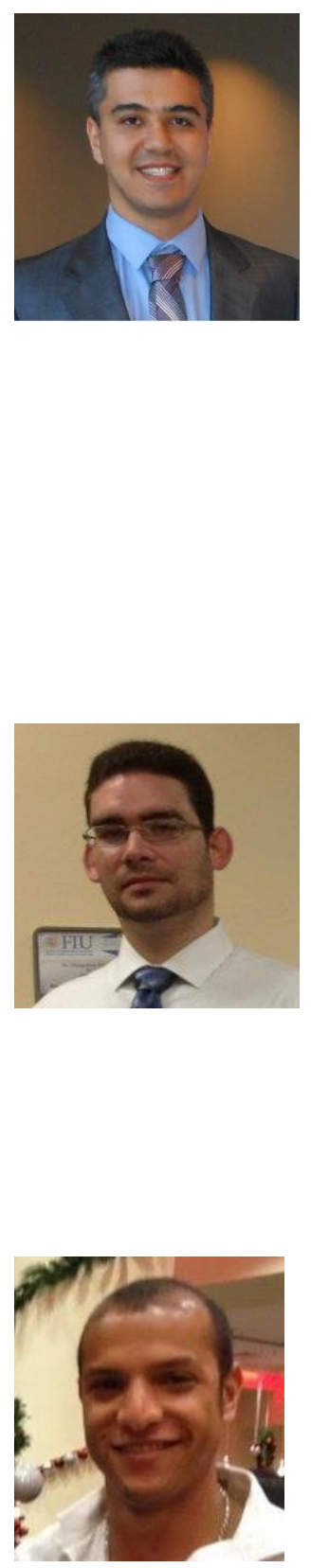

Hadi Fekrmandi received his master's degree in mechanical engineering from the University of Tabriz in 2009. His research topic was nonlinear vibration behavior of cracked structures. He also worked on several projects related to vibration based-SHM during his master thesis. $\mathrm{He}$ is currently working on his Ph.D. dissertation at Florida International University, Mechatronics Research Laboratory. His current research interests include structural health monitoring using piezoelectric and laser vibrometer sensors; machine condition monitoring (MCM) and embedded electronic device development.

Javier Rojas graduated from Florida International University with a Master's Degree in Mechanical Engineering in 2013. His academic focus was on computational numerical methods and applied mathematics with focus on the optimization. His research focus was implementation of SHM with microelectronics and laser Vibrometers.

Jason Campbell is currently an undergraduate, pursuing his Bachelor's degree in Mechanical Engineering at Florida International University, and is scheduled to graduate in the spring of 2015 . His plans for the near future include beginning his senior project and possible internship at Lockheed.
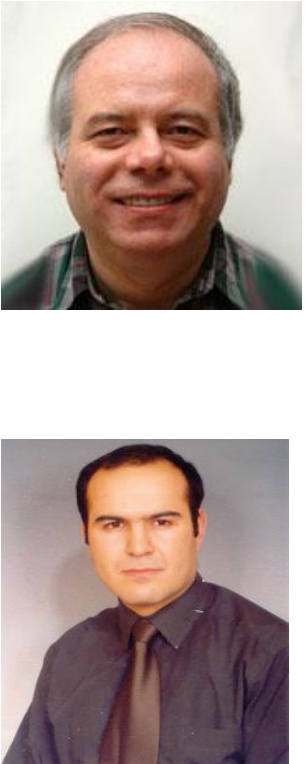

IIbrahim Nur Tansel is a Professor at Department of Mechanical and Materials Engineering, Florida International University in Miami. His research fields are micro machining, tool wear condition monitoring and active structural health monitoring systems using piezoelectric sensors.

Bulent Kaya received his M.S. degree in Design and Manufacturing Engineering from Gebze Institute of Technology in 2002 and Ph.D. degree in Mechanical Engineering from Kocaeli University in 2009. He is currently an Assistant Professor of Industrial Design Engineering, School of Engineering, Erciyes University, Turkey. His research interests are CAD-CAM, tool condition monitoring and unmanned manufacturing systems.

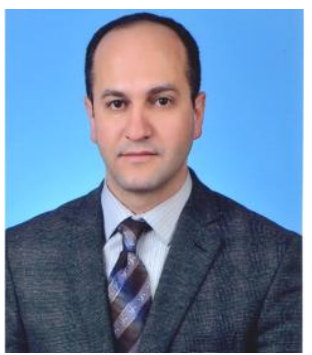

Sezai Taskin received his B.Sc., M.Sc. and Ph.D. degrees in electrical education from the University of Marmara in 1999, 2001 and 2007 respectively. He is currently working at Celal Bayar University's Electrical and Electronics Engineering Department. His current research interests include smart grids, condition monitoring applications and control system design. 